\title{
Microestrutura de pêlos-guarda de felídeos brasileiros: considerações para a identificação de espécies
}

\author{
Ralph Eric Thijl Vanstreels ${ }^{1,4}$, Fernanda do Passo Ramalho ${ }^{2}$ \& Cristina Harumi Adania $^{3}$ \\ ${ }^{1}$ Departmento de Patologia, Faculdade de Medicina Veterinária e Zootecnia, Universidade de São Paulo - USP, \\ Av. Orlando Marques de Paiva, 87, CEP 05009-000 São Paulo, SP, Brasil \\ ${ }^{2}$ Departamento de Genética e Evolução, Universidade Federal de São Carlos - UFSCar, \\ Rua Américo Jacomino Canhoto, 175, 44F, CEP 13564-350 São Carlos, SP, Brasil \\ ${ }^{3}$ Associação Mata Ciliar, Av. Emilio Antonon, 1000, CEP 13212-010 Jundiaí, SP, Brasil \\ ${ }^{4}$ Autor para correspondência: Ralph Eric Thijl Vanstreels, e-mail: ralph_vanstreels@yahoo.com.br
}

VANSTREELS, R.E.T., RAMALHO, F.P. \&ADANIA, C.H. Guard-hair microstructure of Brazilian felids: considerations for species identification. Biota Neotrop.10(1): http://www.biotaneotropica.org.br/v10n1/en/ abstract?article+bn00910012010.

\begin{abstract}
The analysis of cuticle and medulla hair microstructure is a simple and inexpensive technique to identify mammal species for a variety of applications. We studied the guard-hairs of 66 individuals of eight felid species occurring in Brazil (Leopardus colocolo, L.geoffroyi, L.pardalis, L.tigrinus, L.wiedii, Panthera onca, Puma concolor, Puma yagouaroundi), through hair samples collected from anesthetized zoo animals. The microstructure of the guard-hairs was analyzed and described through cuticle impressions and medulla preparations; a blind test was conducted to evaluate the accuracy of species identification. Although distinctive morphological characters could be identified for each species, the subtlety of these characters and the overlap of features among species resulted in a relatively poor accuracy $(75 \%)$. The identification of pairs or trios of species whose hair has similar morphologies (Group A: L. pardalis, L. tigrinus, L. wiedii; Group B: L. colocolo, L. geoffroyi, P. yagouaroundi; Group C: P. concolor, P. onca) significantly improved accuracy (91\%). The identification of Brazilian felids through the microstructure of their hair is challenging and requires careful examination of subtle features, and should be complemented by more accurate techniques and/or be limited mostly to applications where high accuracy is not essential or where a broader taxonomic scale is being evaluated.
\end{abstract}

Keywords: Brazil, Felidae, guard-hair, tricology.

VANSTREELS, R.E.T., RAMALHO,F.P.\&ADANIA, C.H. Microestrutura de pêlos-guarda defelídeos brasileiros: considerações para a identificação de espécies. Biota Neotrop.10(1): http://www.biotaneotropica.org.br/v10n1/ en/abstract?article+bn00910012010.

Resumo: O exame da microestrutura da cutícula e medula dos pêlos é uma técnica simples e de baixo custo para identificar espécies de mamíferos para uma variedade de aplicações. Foram estudados pêlos-guarda de 66 indivíduos de oito espécies de felídeos brasileiros (Leopardus colocolo, L.geoffroyi, L.pardalis, L.tigrinus, L.wiedii, Panthera onca, Puma concolor, Puma yagouaroundi), através de amostras colhidas de animais anestesiados em zoológicos. A microestrutura dos pêlos-guarda foi analisada e descrita através de impressões cuticulares e preparações medulares, e posteriormente foi feito um teste cego para avaliar a acurácia da identificação específica. Embora tenham sido encontrados caracteres morfológicos distintos para cada espécie, a sutileza destes caracteres e sua sobreposição entre as diferentes espécies resultaram em uma acurácia relativamente baixa (75\%). A identificação de pares ou trios de espécies cujos pêlos têm morfologia mais semelhante (Grupo A: L. pardalis, L. tigrinus, L. wiedii; Grupo B: L. colocolo, L. geoffroyi, P. yagouaroundi; Grupo C: P. concolor, P. onca) elevou a acurácia significativamente $(91 \%)$. A identificação dos felídeos brasileiros através da microestrutura de seus pêlos é desafiadora e requer um exame cuidadoso de caracteres sutis, devendo ser apoiada por outras técnicas mais acuradas e/ou ser limitada principalmente às aplicações que não requerem identificação específica ou que trabalhem em escalas taxonômicas mais amplas.

Palavras-chave: Brasil, Felidae, pêlos-guarda, tricologia. 


\section{Introdução}

A identificação da espécie através da avaliação da estrutura microscópica de seus pêlos é uma ferramenta simples e de baixo custo para estudos ecológicos (Chernova 2001, Teerink 2004, Kendall \& McKelvey 2008) e que também tem aplicações na ciência forense (Deedrick \& Koch 2004), arqueologia (Dove \& Perauch 2002), paleontologia (Meng \& Wyss 1997) e na identificação de falsificações têxteis (Hara et al. 1993) ou no monitoramento do comércio ilegal de peles (Tonin et al. 2002). Os pêlos podem ser classificados em dois grupos principais: os subpêlos são mais curtos, finos, ondulados e numerosos, e contribuem à termorregulação e proteção; e os pêlosguarda são mais longos, grossos e menos numerosos, sobressaem-se na pelagem e contribuem principalmente à coloração da pelagem e mecanorrecepção (Teerink 2004). Os pêlos-guarda são mais úteis para a identificação específica através da microestrutura de sua cutícula e medula, que muitas vezes forma padrões morfológicos característicos para cada espécie (Chernova 2001, Teerink 2004).

Apesar da ampla gama de aplicações práticas desta técnica, há relativamente pouca literatura publicada sobre a morfologia dos pêlos dos mamíferos brasileiros, e a maioria dos dados existentes é focada em espécies argentinas que também ocorrem em território brasileiro (Piantanida \& Petriella 1976, Chehébar \& Martins 1989, Férnandez \& Rossi 1998, Vázquez et al. 2000). Informações específicas para a fauna brasileira são escassas e estão fragmentadas (Hess et al. 1985, Müller 1989, Quadros \& Monteiro-Filho, 1998a, 1998b, Quadros 2002, Ingberman \& Monteiro-Filho 2006, Martin et al. 2009), e várias espécies ainda não tiveram suas características tricológicas descritas em detalhe.

Os felídeos neotropicais são um grupo particularmente desafiador em relação à identificação específica pelo exame microscópico de seus pêlos, e nem todas as espécies foram detalhadamente descritas e comparadas entre si (Chehébar \& Martín 1989, Vázquez et al. 2000, Quadros 2002, Miotto et al. 2007). Oito espécies de felídeos selvagens ocorrem no Brasil (Oliveira \& Cassaro 1999): Gato-palheiro Leopardus colocolo (Molina, 1782), Gato-do-mato-grande Leopardus geoffroyi (d'Orbigny \& Gervais, 1844), Jaguatirica Leopardus pardalis (Linnaeus, 1758), Gato-do-mato-pequeno Leopardus tigrinus (Schreber, 1775), Gato-maracajá Leopardus wiedii (Schinz, 1821), Onça pintada Panthera onca (Linnaeus, 1758), Onça parda Puma concolor (Linnaeus, 1771) e Gato-mourisco Puma yagouaroundi (É. Geoffroy Saint-Hilaire, 1803). No presente trabalho apresentaremos a descrição das características microestruturais dos pêlos-guarda destes felídeos a partir de espécimes de cativeiro, e discutiremos a confiabilidade da identificação tricológica aplicada a estas espécies e suas implicações para estudos de campo.

\section{Material e Métodos}

Os pêlos de 66 indivíduos adultos foram analisados: 4 Leopardus colocolo, 9 Leopardus geoffroyi, 9 Leopardus pardalis, 9 Leopardus tigrinus, 9 Leopardus wiedii, 8 Panthera onca, 9 Puma concolor, 9 Puma yagouaroundi. As amostras de pêlos foram colhidas da região escapular de animais cativos em zoológicos brasileiros entre 1996 e 2000, durante procedimentos anestésicos de rotina. A distribuição geográfica dos zoológicos em que foram colhidas as amostras é apresentada na Tabela 1. Os animais foram levados aos zoológicos após terem sido trazidos de vida-livre, porém infelizmente não foi possível obter registros precisos de suas subespécies ou locais de captura; é provável que as amostras tenham incluído diversas subespécies ou populações distintas devido à variedade da distribuição geográfica dos zoológicos que mantinham os animais estudados. Os pêlos foram arrancados manualmente com luvas de látex, e armazenados em envelopes plásticos à temperatura ambiente na Associação Mata Ciliar (Jundiaí-SP, Brasil) por 4 a 8 anos até serem examinados.

De cada amostra individual, foram analisadas três subamostras com cinco pêlos-guarda cada (pêlos-guarda com angulação entre a haste e o escudo; tipo GH2 segundo a classificação proposta por Teerink 2004) num total de 990 pêlos examinados, ou 15 pêlos por indivíduo. Lâminas microscópicas de medula e impressões cuticulares foram preparadas segundo as técnicas descritas por Quadros \& Monteiro-Filho (2006a), utilizando esmalte de unhas comercial como resina para as impressões cuticulares (Maybelline Extra-brilho, Colorama, Ceil, Coml. Exp. Indl. Ltda.). A leitura das lâminas microscópicas foi feita em microscopia de luz, sob aumento de 100-400×. Dois observadores treinados (R.E.T. Vanstreels, F.P. Ramalho) analisaram os pêlos separadamente e elaboraram suas descrições da morfologia microestrutural dos pêlos, e posteriormente compararam suas descrições e montaram uma descrição conjunta com base nos caracteres consensuais. A nomenclatura da microestrutura tricológica ainda não está bem definida e existem abordagens variadas de descrição morfológica, de modo que serão apresentadas ambas as nomenclaturas brasileira (Quadros \& Monteiro-Filho 2006b) e inglesa (Teerink 2004).

Após a descrição detalhada da morfologia microestrutural dos pêlos foram conduzidos testes cegos para determinar a acurácia da identificação específica. Decorridos 14 meses da descrição morfológica, um observador treinado (R.E.T. Vanstreels) sorteou 40 indivíduos (dentre os 66 indivíduos analisados originalmente) e preparou novas lâminas microscópicas com cinco pêlos-guarda de cada amostra. Trabalhando sem conhecer as espécies às quais os pêlos examinados pertenciam e utilizando as descrições dos caracteres morfológicos microestruturais elaboradas previamente, o observador chegou a um

Tabela 1. Distribuição geográfica dos zoológicos em que foram colhidas as amostras de pêlos.

Table 1. Geographic distribution of the zoos where the samples were collected.

\begin{tabular}{|c|c|c|c|c|c|c|c|}
\hline & Amazonas & Pará & $\begin{array}{l}\text { Distrito } \\
\text { Federal }\end{array}$ & $\begin{array}{l}\text { Minas } \\
\text { Gerais }\end{array}$ & $\begin{array}{c}\text { São } \\
\text { Paulo }\end{array}$ & Paraná & $\begin{array}{c}\text { Rio Grande } \\
\text { do Sul }\end{array}$ \\
\hline L.colocolo & - & - & 1 & 1 & 2 & - & - \\
\hline L.geoffroyi & - & - & - & - & 1 & - & 8 \\
\hline L.pardalis & - & - & - & - & 9 & - & - \\
\hline L.tigrinus & - & - & 2 & 3 & 4 & - & - \\
\hline L.wiedii & 3 & - & - & - & - & 6 & - \\
\hline P.onca & - & 1 & 1 & - & 6 & - & - \\
\hline P.concolor & 1 & 2 & - & - & 6 & - & - \\
\hline P.yagouaroundi & - & - & - & 4 & 5 & - & - \\
\hline Total & 4 & 3 & 4 & 8 & 33 & 6 & 8 \\
\hline
\end{tabular}


diagnóstico de espécie para cada amostra e estes resultados foram então comparados com os registros de espécie das amostras originais.

\section{Resultados}

À semelhança do descrito para outros carnívoros (Quadros 2002, Teerink 2004), nas espécies estudadas o terço distal da haste dos pêlos-guarda foi considerada a melhor região para a identificação, com as características estruturais mais distintivas. Todas as espécies apresentaram variações do padrão pavimentoso losângico intermediário nas regiões proximal e média da haste, assim como um padrão pavimentoso ondeado transversal ornamentado no escudo, de modo que essas regiões foram consideradas de pouco valor diagnóstico. As características microestruturais cuticulares e medulares são apresentadas na Figura 1 e nas Tabelas 2 e 3.

As espécies foram separadas em três grupos principais (Grupos A-C), agrupando aquelas em que os pêlos-guarda apresentaram características microestruturais mais semelhantes. O Grupo A inclui três espécies (L. pardalis, L. tigrinus, $L$. wiedii) que são diferenciadas pela largura das escamas e sua projeção aos bordos. L.wiedii possui as escamas mais estreitas e que mais se projetam aos bordos. Em L.tigrinus, as escamas são estreitas (porém não tanto quanto em L.wiedii) e não se projetam aos bordos. Já as escamas L.pardalis possuem largura intermediária e se projetam moderadamente aos bordos. O Grupo B inclui três espécies (L. colocolo, L. geoffroyi, P. yagouaroundi) que

$L W$

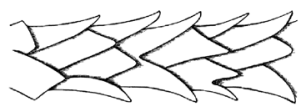

$L t$

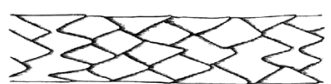

$L p$

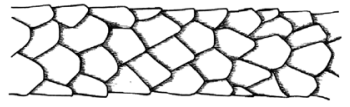

Lc

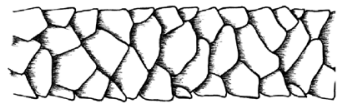

Py

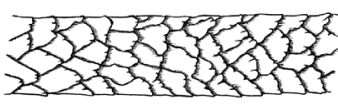

$\operatorname{Lg}$

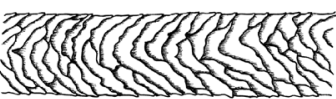

PC

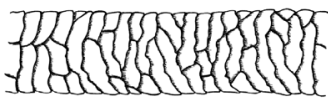

Po

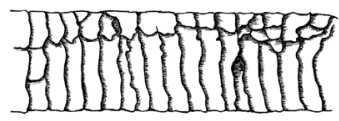

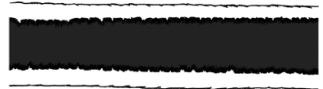
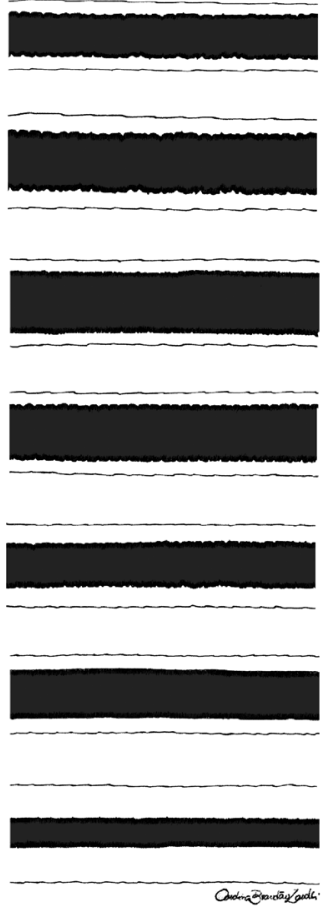

Figura 1. Morfologia cuticular (esquerda) e medular (direita) do terço distal da haste de pêlos-guarda em felídeos brasileiros. Leopardus wiedii (Lw), Leopardus tigrinus (Lt), Leopardus pardalis (Lp), Leopardus colocolo (Lc), Puma yagouaroundi (Py), Leopardus geoffroyi (Lg), Puma concolor (Pc), Panthera onca (Po).

Figure 1. Cuticle (left) and medulla (right) morphology of the upper shaft of guard-hair from Brazilian felids. Leopardus wiedii (Lw), Leopardus tigrinus (Lt), Leopardus pardalis (Lp), Leopardus colocolo (Lc), Puma yagouaroundi (Py), Leopardus geoffroyi (Lg), Puma concolor (Pc), Panthera onca (Po). são diferenciadas pela largura e orientação de suas escamas. Em P.yagouaroundi, há um gradiente de variação entre escamas losângicas largas e um padrão mosaico, sempre com as bordas ligeiramente ornamentadas. L.colocolo possui uma ampla gama de variações em seu padrão cuticular, suas escamas losângicas acentuadamente largas podem ser confundidas às vezes com o padrão ondeado transversal, porém em geral haverá uma região em que as escalas mais losângicas formam uma linha mais central (assemelhando-se ao padrão streaked descrito por Teerink 2004, porém com as escamas tendo formato de pétalas e bordos bem definidos). L.geoffroyi pode apresentar um padrão ligeiramente semelhante ao de L.colocolo devido à orientação de suas escamas, porém tipicamente, em alguma região, apresentará um padrão ondeado oblíquo duplo, em que suas largas escamas se orientam de maneira a formar um desenho em $\mathrm{V}$ ao longo da haste. O Grupo C inclui os dois maiores felídeos estudados ( $P$. concolor, $P$. onca), que apresentam microestrutura cuticular muito semelhantes, porém podem ser facilmente diferenciadas com base na largura de sua medula. A medula dos pêlos de P.concolor possui uma largura superior a $2 / 3$ da largura do pêlo, enquanto que em $P$.onca a medula nunca ultrapassa os $2 / 3$ da largura do pêlo.

Em algumas espécies, foram notadas características macroscópicas dos pêlos-guarda que ajudam a identificação da espécie: L.colocolo tem um comprimento de pêlos altamente heterogêneo (alguns pêlos podem ser particularmente mais longos do que em todas as outras espécies, atingindo $8-10 \mathrm{~cm}$ enquanto as demais espécies têm tipicamente $3-5 \mathrm{~cm}$ ), a haste dos pêlos tende a ser muito longa e o escudo proporcionalmente muito curto, quase sempre com uma angulação de $45^{\circ}$ entre haste e escudo; P.onca tem um comprimento de pêlos muito homogêneo, com os pêlos sendo retos e consistentemente mais curtos que nas demais espécies (raramente mais longo que $2 \mathrm{~cm}$ ), o que ajuda a diferenciação em relação a P.concolor (comprimento típico de 2-4 cm). Nas demais espécies, os pêlos-guarda variam entre $3-5 \mathrm{~cm}$ de comprimento e têm colorações variando em tons de branco, amarelo-dourado, marrom e preto; com um padrão de coloração homogênea ou agouti (alternância longitudinal de uma faixa de pigmento feomelânico amarelo-dourado entre duas faixas de pigmento eumelânico marrom-preto).

Baseado nestas descrições, o teste cego revelou que a identificação da espécie teve uma acurácia de 75,0 \pm 14,9\% (Média \pm Desvio Padrão), enquanto que a identificação do grupo de espécies teve acurácia de 91,3 $\pm 11,0 \%$ (Qui-Quadrado; $X_{2}=7,530$; $\mathrm{DF}=1 ; \mathrm{P}=0,003)$. Não houve diferenças significativas entre grupos na acurácia para o diagnóstico da espécie (Qui-Quadrado; $\mathrm{X}_{2}=3,236$; $\mathrm{DF}=2 ; \mathrm{P}=0,198$ ) ou do grupo (Qui-Quadrado; $\mathrm{X}_{2}=2,231 ; \mathrm{DF}=2$; $\mathrm{P}=0,328)$.

\section{Discussão}

Há controvérsia sobre a confiabilidade da identificação de espécies através da microestrutura de seus pêlos. Vilchis et al. (2005) analisaram características estruturais dos pêlos de quatorze mamíferos tropicais, e concluíram que características morfológicas quantitativas não são confiáveis para a identificação destas espécies, mas que características qualitativas como a morfologia medular são altamente específicas. Outros autores aplicaram microscopia eletrônica de transmissão para desenvolver guias de identificação baseados em mensurações de largura medular (Moore \& Braun 1983) ou características qualitativas (Hess et al. 1985). De Marinis e Asprea (2006) utilizaram testes cegos para determinar a acurácia na identificação de dez espécies de ungulados europeus através da análise da morfologia cuticular e medular, e obtiveram $98 \%$ de acurácia para identificar a espécie, $100 \%$ para a faixa etária e $97 \%$ para a estação do ano. 
Tabela 2. Morfologia cuticular do terço distal da haste de pêlos-guarda em felídeos brasileiros.

Table 2. Cuticular morphology on the upper shaft of guard-hair from Brazilian felids.

\begin{tabular}{|c|c|c|}
\hline Grupo A & Nomenclatura brasileira & English nomenclature \\
\hline L. wiedii & Imbricada, folidácea, muito estreita & Longitudinal, very elongate petal, smooth, distant \\
\hline L. tigrinus & Pavimentosa, losângica, estreita & Longitudinal, narrow diamond petal, smooth, distant \\
\hline L. pardalis & Imbricada, folidácea, intermediária & Longitudinal, broad petal, smooth, distant \\
\hline \multicolumn{3}{|l|}{ Grupo B } \\
\hline L. colocolo & Pavimentosa, losângica, muito larga & Intermediate, very broad diamond petal, smooth, distant \\
\hline P. yagouaroundi & $\begin{array}{l}\text { Pavimentosa, losângica largo ou mosaico, levemente } \\
\text { ornamentada }\end{array}$ & Intermediate, broad diamond petal or mosaic, rippled, distant \\
\hline L. geoffroyi & Pavimentosa, ondeada, oblíqua dupla & Intermediate, regular V-shaped wave, smooth, near \\
\hline \multicolumn{3}{|l|}{ Grupo C } \\
\hline P. concolor & $\begin{array}{l}\text { Pavimentosa, ondeada, transversal, lisa, contínua, } \\
\text { escamas com largura intermediária }\end{array}$ & Transversal, regular wave, smooth, near \\
\hline P. onca & $\begin{array}{l}\text { Pavimentosa, ondeada, transversal, lisa, contínua, } \\
\text { escamas largas }\end{array}$ & Transversal, regular wave, smooth, distant \\
\hline
\end{tabular}

Tabela 3. Morfologia medular da haste de pêlos-guarda em felídeos brasileiros.

Table 3. Medullar morphology on the shaft of guard-hair from Brazilian felids.

\begin{tabular}{|c|c|c|}
\hline Grupo A & Nomenclatura brasileira & English nomenclature \\
\hline $\begin{array}{l}\text { L. wiedii } \\
\text { L. tigrinus } \\
\text { L. pardalis } \\
\end{array}$ & $\begin{array}{l}\text { Trabecular, fimbriada, largura medular com mais de } \\
\text { 2/3 da largura do pêlo }\end{array}$ & $\begin{array}{l}\text { Multicellular, intermediate, scalloped, medullar } \\
\text { width larger than } 2 / 3 \text { of hair width }\end{array}$ \\
\hline \multicolumn{3}{|l|}{ Grupo B } \\
\hline $\begin{array}{l}\text { L. colocolo } \\
\text { P. yagouaroundi } \\
\text { L. geoffroyi }\end{array}$ & $\begin{array}{l}\text { Trabecular, fimbriada, largura medular com mais de } \\
\text { 2/3 da largura do pêlo }\end{array}$ & $\begin{array}{l}\text { Multicellular, intermediate, scalloped, medullar } \\
\text { width larger than } 2 / 3 \text { of hair width }\end{array}$ \\
\hline \multicolumn{3}{|l|}{ Grupo C } \\
\hline P. concolor & $\begin{array}{l}\text { Trabecular, íntegra, largura medular com mais de } 2 / 3 \\
\text { da largura do pêlo }\end{array}$ & $\begin{array}{l}\text { Multicellular, intermediate, straight, medullar width } \\
\text { larger than } 2 / 3 \text { of hair width }\end{array}$ \\
\hline P. onca & $\begin{array}{l}\text { Trabecular, fimbriada, largura medular com menos de } \\
2 / 3 \text { da largura do pêlo }\end{array}$ & $\begin{array}{l}\text { Multicellular, intermediate, scalloped, medullar } \\
\text { width thinner than } 2 / 3 \text { of hair width }\end{array}$ \\
\hline
\end{tabular}

Os felídeos neotropicais são um grupo taxonômico particularmente difícil de identificar por análises tricológicas. Chehébar \& Martín (1989) estudaram mamíferos argentinos e concluíram que os pequenos felinos estudados (Leopardus colocolo, L.geoffroyi, L.jacobitus - Cornalia, 1865) não podem ser diferenciados entre si através da morfologia de seus pêlos. Vázquez et al. (2000) estudaram os pêlos de carnívoros argentinos, e concluíram que a morfologia dos felídeos era excessivamente similar para permitir a diferenciação ao nível de espécies, e prefiriu agrupá-los em dois grupos de espécies de morfologia tricológica similar (Pequenos felídeos e lontra sulamericana: Leopardus colocolo, Leopardus geoffroyi, Leopardus wiedii, Lontra longicaudis - Olfers, 1818; Grandes felinos: Panthera onca, Puma concolor). Miotto et al. (2007) encontraram completa concordância nos resultados de análises tricológicas e métodos biomoleculares para identificar pêlos de carnívoros em fezes coletadas em campo (Cerdocyon thous - Linnaeus, 1766, Leopardus pardalis, Puma concolor, P.yagouaroundi). Quadros (2002) apresentou as características morfológicas dos pêlos de onze espécies de carnívoros brasileiros, e sugere que os felídeos estudados (Leopardus pardalis, L.tigrinus, L.wiedii, Puma concolor, P. yagouaroundi) podem ser diferenciados ao nível de espécie, embora reconheça que a identificação é muito sutil e de fácil confusão.

Nossas descrições da microestrutura dos pêlos das espécies estudadas propõem que existem de fato algumas diferenças sutis entre as espécies de felídeos que ocorrem no território brasileiro. A diferenciação, no entanto, é bastante sutil e a existência de sobreposições de padrões morfológicos entre espécies resulta em uma acurácia limitada (75\%), insuficiente para a maioria das aplicações científicas. É possível agrupar pares ou trios de espécies cujos pêlos têm morfologia mais semelhante, e o diagnóstico ao nível destes grupos é consideravelmente superior (91\%), porém ainda é insuficiente para aplicações científicas mais rigorosas.

Por outro lado, os baixos custos e requerimentos de equipamentos desta técnica ainda são grandes atrativos para justificar seu uso em estudos aplicados e com finalidades de monitoramento populacional, especialmente quando a diferenciação ao nível específico não é indispensável. A identificação da família Felidae ou de um grupo de espécies tricologicamente mais semelhantes podem ser suficientes para estudos que trabalhem em uma escala taxonômica mais ampla, ou que busquem excluir os felídeos de suas análises. Para identificação da espécie em amostras de fezes, a baixa acurácia possivelmente pode 
ser melhorada para níveis mais satisfatórios se o exame tricológico for complementado com observações de campo como rastros ou pegadas, microhábitat no local de coleta das fezes e morfologia fecal (Becker \& Dalponte 1999, Chame 2003), embora também existam críticas à acurácia destas abordagens de campo (Miotto et al. 2007). Para grandes números de amostras, a tricologia microscópica pode servir ainda como ferramenta de triagem para identificar generalidades taxonômicas dos pêlos estudados, enquanto técnicas biomoleculares podem ser aplicadas em uma segunda etapa para identificar com maior acurácia as espécies envolvidas.

\section{Agradecimentos}

Agradecemos à Associação Mata Ciliar e a todos os zoológicos nos quais as amostras foram coletadas. Somos imensamente gratos a Carolina Brandão Zanelli pela assistência artística.

\section{Referências}

BECKER, M. \& DALPONTE, C.J. 1999. Rastros de mamíferos silvestres brasileiros: um guia de campo. Universidade de Brasília, Brasília.

CHAME, M. 2003. Terrestrial mammal feces a morphometric summary and description. Mem. Inst. Oswaldo Cruz 98(suppl. 1):71-94.

CHEHÉBAR, C. \& MARTÍN, S. 1989. Guía para el conocimiento microscópico de los pelos de los mamíferos de la Patagonia. Doñana Acta Vertebr. 16(2):247-291.

CHERNOVA, O.F. 2001. Architectonics of the medulla of guard hair and its importance for identification of taxa. Doklady Biol. Sci. 376(1-6):81-85.

DEEDRICK, D.W. \& KOCH, S.L. 2004. Microscopy of hair part II: a practical guide and manual for animal hairs. Forensic Sci. Comm. 6(3): http://www. fbi.gov/hq/lab/fsc/backissu/july2004/research/2004_03_research02.htm (último acesso em 12/11/2009)

DOVE, C.J. \& PEURACH, S.C. 2002. Microscopic analysis of feather and hair fragments associated with human mummified remains from Kagamil Island, Alaska. Ethnogr. Ser. 20:51-62.

FERNÁNDEZ, G.J. \& ROSSI, S.M. 1998. Medullar type and cuticular scale patterns of hairs of rodents and small marsupials from the Monte Scrubland (San Luis Province, Argentina). Mastozool. Neotrop. 5(2):109-116.

HARA, A., HIRAI, I. \& GUNJI, T. 1993. Morphology of Mink fur and fake furs. J. Tex. Mach. Soc. Japan 39(4):82-87.

HESS, W.M., FLINDERS, J.T., PRITCHETT, C.L. \& ALLEN, J.V. 1985 Characterization of hair morphology in families Tayassuidae and Suidae with scanning electron microscopy. J. Mammal. 66(1):75-84.

INGBERMAN, B. \& MONTEIRO FILHO, E.L.A. 2006. Identificação microscópica dos pêlos das espécies brasileiras de Alouatta Lacépéde, 1799 (Primates, Atelidade Alouattinae). Arq. Mus. Nac. 64(1):61-71.

KENDALL, K.C. \& MCKELVEY, K.S. 2008. Hair collection. In Noninvasive survey methods for carnivores (R.A. Long, P. MacKay, J. Ray, \& W. Zielinski, eds). Island Press, Washington.

MARINIS, A.M. \& ASPREA, A. 2006. Hair identification key of wild and domestic ungulates from southern Europe. Wildl. Biol. 12(3):305-320.

MARTIN, P.S., GHELER-COSTA, C. \& VERDADE, L.M. 2009. Microestruturas de pêlos de pequenos mamíferos não-voadores: chave para identificação de espécies de agroecossistemas do estado de São Paulo, Brasil. Biota Neotrop. 9(1):233-241.

MENG, J. \& WYSS, A.R. 1997. Multituberculate and other mammal hair recovered from Palaeogene excreta. Nature 385(6618):712-714.

MIOTTO, R.A., CIOCHETTI, G., RODRIGUES, F.P. \& GALETTI Jr., P.M. 2007. Identification of pumas (Puma concolor (Linnaeus, 1771)) through faeces: a comparison between morphological and molecular methods. Braz. J. Biol. 67(suppl. 4):963-965.

MOORE, D.W. \& BRAUN, J.K. 1983. Keys to the hairs of the families Soricidae, Vespertilionidae, and Muridae within Tennessee. J. Tennessee Acad. Sci. 58(3-4):40-43.

MÜLLER, M.V.Y. 1989. Microestrutura de pelos de mamíferos: métodos de análise e sua aplicação na identificação de algumas espécies do Estado do Paraná, Brasil. Dissertação de Mestrado, Universidade Federal do Paraná, Curitiba.

OLIVEIRA, T.G. \& CASSARO, K. 1999. Guia de identificação dos felinos brasileiros. Sociedade de Zoológicos do Brasil, São Paulo.

PIANTANIDA, M. \& PETRIELLA, A.M. 1976. Estudio morfológico de algunas especies de roedores de la Provincia de Buenos Aires con el microscopio electrónico de barrido. Physis 35(90):105-124.

QUADROS, J. 2002. Identificação microscópica de pêlos de mamíferos brasileiros e sua aplicação no estudo da dieta de carnívoros. Dissertação de Mestrado, Universidade Federal do Paraná, Curitiba.

QUADROS, J. \& MONTEIRO-FILHO, E.L.A. 1998a. Effects of digestion, putrefaction and taxidermy processes on Didelphis albiventris hair morphology. J. Zool. 244(3):331-334.

QUADROS, J. \& MONTEIRO-FILHO, E.L.A. 1998b. Morphology of different hair types of Didelphis albiventris and its usage in hair identification. Cien. Cult. 50(5):382-385.

QUADROS, J. \& MONTEIRO-FILHO, E.L.A. 2006a. Coleta e preparação de pêlos de mamíferos para identificação em microscopia ótica. Rev. Bras. Zool. 23(1):274-278.

QUADROS, J. \& MONTEIRO-FILHO, E.L.A. 2006b. Revisão conceitual, padrões microestruturais e proposta nomenclatória para os pêlos-guarda de mamíferos brasileiros. Rev. Bras. Zool. 23(1):279-292.

TEERINK, B.J. 2004. Hair of West European mammals: atlas and identification key. Cambridge University Press, Cambridge.

TONIN, C., BIANCHETTO, M., VINEIS, C. \& BIANCHET, M.F. 2002. Differentiating fine hairs from wild and domestic species: Investigations of Shatoosh, Yangir, and Cashmere fibers. Tex. Res. J. 72(8):701-705.

VÁZQUEZ, D.E., PEROVIC, P.G. \& OLSEN, A.A. 2000. Patrones cuticulares y medulares de pelos de mamíferos del noroeste argentino (Carnivora y Artiodactyla). J. Neotrop. Mammal. 7(2):131-147.

VILCHIS, O.M., MORALES, C.G., RODRÍGUEZ, R.R., MARTÍN, A.D.H.S., CASTRO, J.P.M., REYES, U.A. \& GARCÍA, A.I.O. 2005. Variación intraespecífica e individual de los pelos de mamíferos del Estado de México: implicaciones en la identificación interespecífica. Cien. Ergo Sum. 12(3):264-270. 